\title{
Design and Analysis of Printed Rectangular Micro Strip Antenna on Ferrite Material
}

\author{
Amandeep Kour \\ Assistant Professor \\ Department of computer science \& Engineering, \\ Mahant Bachittar Singh College of Engineering \& Technology, Babliana \\ Jammu (J\&K), India
}

\begin{abstract}
The study of Micro strip patch antenna is proposed by using artificial neural network. The geometry modelled for the study is rectangular patch type which will be mounted on ferrite substrate. The artificial neural network is used to study the modelled micro strip antenna by using synthesis and analytical approach. The relation between different parameter and dimensions of micro strip antenna are obtained. The patch dimension which is denoted by $(\mathrm{W}, \mathrm{L})$ are dependent of various input variable that can be further divided as resonant frequency $\left(f_{r}\right)$, height of dielectric substrate(h) and the dielectric constant of dielectric material $\left(\varepsilon_{\mathrm{X}}, \varepsilon_{\mathrm{y}}\right)$. The results which are obtained using artificial neural network for the designed antenna are comparable with the result available in the patch antennas literature, theoretically as well as experimentally. The designed procedure is divided into synthesis and analysis which are forward and reverse side respectively. The designed procedure is used and ANN can be applied to the other geometry of antenna in general approach.
\end{abstract}

Index Terms - Artificial neural networks, Micro strip antenna, reverse modelling, radial basis function, Ferrite material.

\section{INTRODUCTION}

The low profile antennas are required for high- performance spacecraft, satellite and missiles application, aircraft where ease of installation, cost, weight, performance and aerodynamic profile are bigger constraints. Along with these, the wireless \& mobile radio communication and any other commercial \&government application where identical specifications are used. Micro-strip antennas can be effectively used to meet this type of requirement.

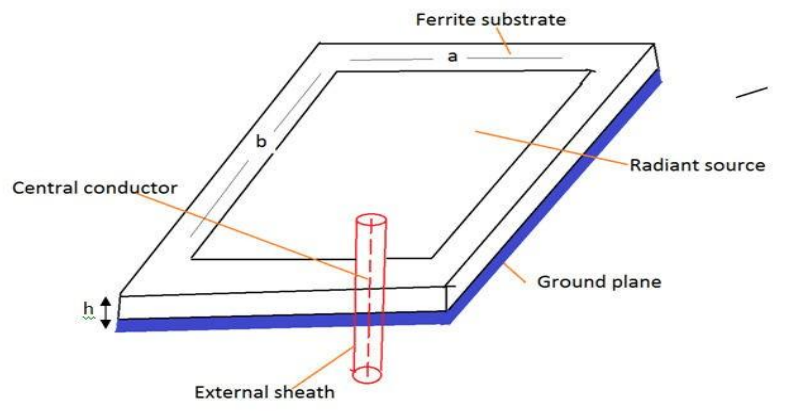

The application of magnetic materials has been increasing day by day because of high frequency requirement; one of the magnetic materials which can be used in single as well as polycrystalline for both types is ferrite. It can be very useful because of its properties over normal dielectric material \& thus it is very useful for the application in microwave antenna. The dielectric constant of ferrite material is high, that's why, the use of ferrite makes this antenna very low profile, suitable with planar as well as for non planar surfaces, easy and low priced to manufacture by using printed circuit technology [1-3].

Since eighties, ANN has been extremely researched for various applications and adaptive controller is one of them. In the beginning of nineties, there is use of artificial neural network while dealing with microwaves. It used in active and passive component modelling, for micro strip antennas modelling, in optimizing microwave devices and for impedance matching etc. Now days, the engineers who are working in the field of microwave are trying very hard to simplify a very complex, difficult designing of microwave circuit, which is very time consuming. ANN are playing very important role in today's world for making self control and smart electronic device. Since they can be cloned and can work like a human brain if properly calibrated according to the desired application. Since ANN works on the different concepts of biological world, In biological world we know that different systems are comprised of many subsystems or small units which may be linearly or non linearly interrelated likewise, we can say that ANN is also formed by the combination of different nonlinear subsystem and the units which are called neurons. Neurons are kept according to the definite structure in which they are arranged in parallel sequence in form of layer and in which, each neuron are denoted by carrying some weight or values. These values are changed or depend on different application for the output.

Since neural network is working like brain therefore it shows the ability to learn from it past sequences of information for particular output. So ANN shows very high speed of operation. According to the literature, ANN is also used in the microwave engineering for designing various types of antennas which vary in the terms of shape \& size. For Example, either it may be equilateral triangle, circular and rectangular patch antennas [4-7].The analysis of the above frequency which ultimately depends on the dielectric of the material as well as the geometric structure.

Fig.1. Geometry of Rectangular Antenna for single patch 
In this paper, the model is design for finding out the dielectric constants of the dielectric material ( $\varepsilon x, \varepsilon y)$ which is ultimately decided according to the different parameter like dielectric substrate height $(\mathrm{h})$, dimensions of patch of rectangular micro strip antennas (W,L), and the resonant frequency (fr) (Fig.1). The value of different dielectric constant and the parameter are calculated using theoretical formulas which are related to the given concept. In this simulation, we have focused on two aspects of design of antenna which are resonant frequency of antenna and various condition to be required for getting the pattern which are formed by good radiation. By using reverse modelling of methodology, the other parameter of antenna design called dimension of patch of rectangular micro strip antenna. The overall formulated and simulated models are very easy for application purpose and also less complex for the engineers to predict $\&$ find out different values of resonant frequency $\&$ patch dimensions. [8-11].

\section{RECTANGULAR MICROSTRIP ANTENNAS}

The dimensions of rectangular micro strip antenna are length $\mathrm{L}$, and width $\mathrm{W}$ which is made by rectangular patch. The rectangular micro strip antenna are mounted on ground plane with thickness of substrate is (h) and dielectric constant is $\left(\varepsilon_{\mathrm{X}}, \varepsilon_{\mathrm{y}}\right.$.) Generally the range of dielectric constant are used for antenna theory is $2.2 \leq \varepsilon r \leq 12$. However, for low Profile antenna and reduction of surface wave, the high dielectric constant is desirable.

For the present paper, the selection of dielectric constant is an important factor in an isotropic structure for doing all works. Now, we are changing denotation of different parameter for anisotropic type of ferrite structure as compared to isotropic. In anisotropic, we will use heff effective spacing instead of spacing parameter (h) and for other parameter which is called geometric mean $(\varepsilon \mathrm{m})$ is used in place of $(\varepsilon \mathrm{r})$ dielectric constant.

$$
h_{\text {eff }}=\sqrt{\frac{\varepsilon_{y}}{\varepsilon_{m}}} h \text { and } \varepsilon_{m}=\sqrt{\varepsilon_{x} \varepsilon_{y}}
$$

$$
\varepsilon_{\text {eff }}=\frac{\varepsilon_{m}+1}{2}+\frac{\varepsilon_{m}-1}{2}\left[1+12 \frac{h_{\text {eff }}}{W}\right]^{\frac{-1}{2}}
$$

Width and Length- defined pattern can be done for finding out the resonant

$$
\begin{gathered}
W=\frac{c}{2 f_{r}} \sqrt{\frac{2}{\varepsilon_{m}+1}} \\
L=\frac{c}{2 f_{r} \sqrt{s_{\text {eff }}}}-2 \Delta L \\
\frac{\Delta L}{h}=0.412 \frac{\left(\varepsilon_{\text {eff }}+0.3\right)\left(\frac{W}{h}+0.264\right)}{\left(\varepsilon_{\text {eff }}+0.258\right)\left(\frac{W}{h}+0.8\right)}
\end{gathered}
$$

Due to the fringing effects here $\Delta L$ is the extension of the Length

\section{NEURAL NETWORK ANALYSIS}

In Artificial neural network models, Radial Basis Function (RBF) networks are used in this work [12-16].

A. The forward side and reverse side

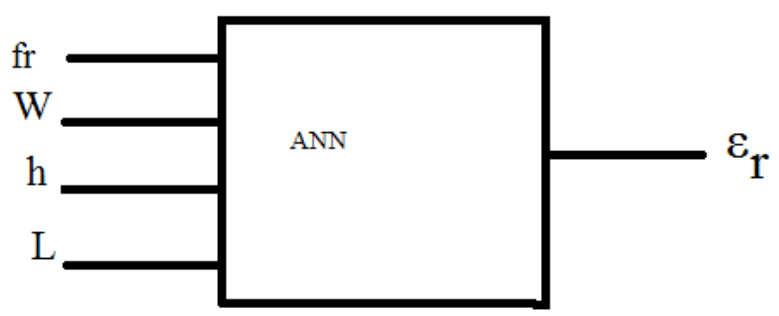

Fig.2. Forward side ANN model

In fig. $2 \& 3$, the ANN synthesis \& ANN analysis input's \& output's quantities are shown. We are representation, our study in two different parts, one is synthesis and another is analysis. For the analysis part all the terminology wills remains same expect the dielectric constant. The output of ANN is dielectric constant is obtained for best selection of dimension of patch and the resonant frequency at the input side

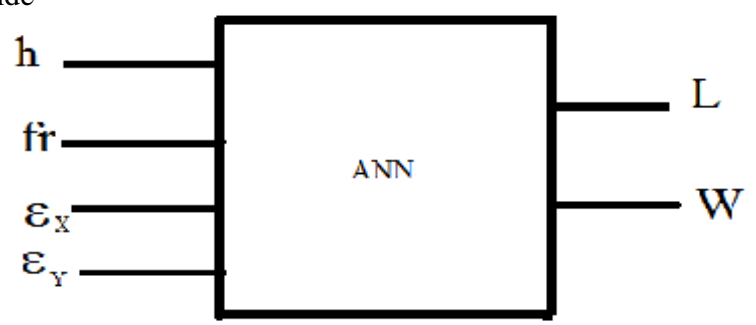

Fig.3. Reverse side ANN model

substrate.

Where, h: height of the dielectric

$\mathrm{f}_{\mathrm{r}}$ : resonant frequency of the antenna.

$\mathrm{L}$ : length of the patch

$\mathrm{W}$ : width of the patch

$\boldsymbol{\varepsilon}_{\mathrm{x}}, \boldsymbol{\varepsilon}_{\mathrm{y}}$ : permittivity in the $\mathrm{x}$ and $\mathrm{y}$ directions respectively of the ferrite material 


\section{B. Radial Basis Function Network}

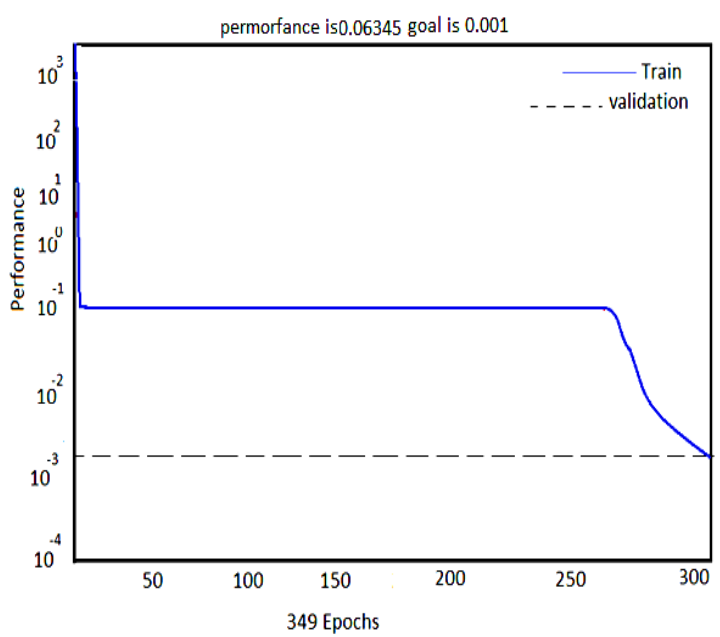

Fig. 4. Training Response at 349 Epochs

RBF Network are those network in which neural network of feed forward consist of single layer which is hidden which uses active function of radial basis for hidden neurons. For modelling of microwave systems, RBF network are applicable. The structure of RBF network is shown Fig. 4.The centre of radial basis activation function is represented by cij and standard deviation is represent by $\lambda_{i j}$ The multiquadratic and Gaussian function is used commonly for radial basis activation function. The $\mathrm{y}_{i}$ is given by, the total input to the hidden neuron $i^{\text {th, }}$ where input $\mathrm{x}$ is given

$$
Y_{i}=\sqrt{\sum_{j=1}^{n}\left(\frac{x_{j}-c_{i j}}{\lambda_{i j}}\right)^{2}} \quad, \mathrm{i}=1,2,3 \ldots \ldots \ldots . . \mathrm{N}
$$

The number of hidden neurons $\mathrm{N}$. The output value is for $i^{\text {th }}$ hidden neuron $\mathrm{Zij}=(\mathrm{i})$, where radial basis function is $\sigma(\gamma)$. Finally, we can calculated the radial basis function output from hidden neurons as

$$
\sigma Y
$$

$$
y_{k}=\sum_{i=0}^{N} w_{k i} z_{k i}
$$

Where, the weight of the link between the $i^{\text {th }}$ neuron of the hidden layer and the $k^{\text {th }}$ neuron of the output layer is termed as $w k i$. The RBF network include training parameters of $w$ as $w k 0, w k i, c i j, \lambda i j, \mathrm{k}=1,2, \ldots \mathrm{m}, \mathrm{i}=1,2, \ldots \mathrm{N}, \mathrm{j}=1,2, \ldots \mathrm{n}$.

\section{Neural Networks Structures}

We can choose the spread value as 0.01 , for getting the best accuracy in radial basis function network. For getting the specific result of desired problem, the RBF was trained with the different types samples which are 60 in numbers and it has been also tested for around 20 samples $\&$ from the result we are satisfying that it is giving permissible results. There are 4 inputs and 2 outputs for the synthesis artificial neural network and 3 inputs and 1 output for analysis artificial neural network. The data which we have used for the analysis as well as synthesis, artificial neural network are taken from the previous results of experimental [13-15] \& computer program which are available with us. The test samples are also taken from previous sample.

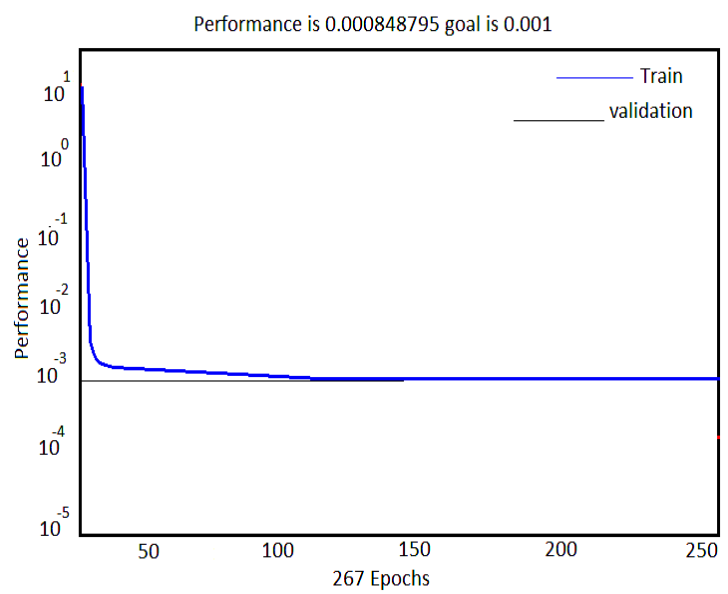

Fig.5. Training Response at 267 Epochs 


\section{RESULTS}

From the above results which are shown in the Tables 1 and 2 . W can say that the radial basis function gives approximate value which is very near to the desired target values. For an isotropic material $(\varepsilon x=\varepsilon y)$ the results of the synthesis and analysis artificial neural network and giving the accurate optimized value when compared with the targets.

TABLE 1

ARTIFICIAL NEURAL NETWORK ANALYSIS RESULTS \& COMPARISON WITH TARGET

\begin{tabular}{|l|l|c|c|c|c|c|}
\hline $\mathbf{h}(\mathbf{c m})$ & & $\mathbf{f r}(\mathbf{G H z})$ & W-target $(\mathbf{c m})$ & W-RBF $(\mathbf{c m})$ & L-target $(\mathbf{c m})$ & L-RBF $(\mathbf{c m})$ \\
\hline 0.1752 & 17.90 & 11.00 & 0.7128 & 0.73850 & 0.35151 & 0.38232 \\
\hline 0.1752 & 17.90 & 10.70 & 0.7334 & 0.73850 & 0.36210 & 0.38232 \\
\hline 0.1752 & 17.90 & 10.60 & 0.7405 & 0.73851 & 0.36577 & 0.38232 \\
\hline 0.1752 & 17.90 & 9.27 & 0.8546 & 0.73982 & 0.42285 & 0.38271 \\
\hline 0.1752 & 17.90 & 9.07 & 0.8713 & 0.74181 & 0.43301 & 0.38346 \\
\hline 0.1752 & 17.90 & 7.00 & 1.1506 & 1.07042 & 0.57655 & 0.58056 \\
\hline 0.1752 & 17.90 & 7.00 & 1.1506 & 1.07042 & 0.57655 & 0.58056 \\
\hline 0.1752 & 17.90 & 11.00 & 0.7128 & 0.73850 & 0.35151 & 0.38232 \\
\hline 0.2642 & 17.90 & 11.00 & 0.6810 & 0.73850 & 0.33517 & 0.38232 \\
\hline 0.2752 & 17.90 & 9.55 & 0.7871 & 0.73850 & 0.38739 & 0.38232 \\
\hline 0.1662 & 16.95 & 9.50 & 0.8114 & 0.73850 & 0.40048 & 0.38232 \\
\hline 0.1662 & 16.95 & 11.30 & 0.6847 & 0.73850 & 0.33528 & 0.38232 \\
\hline 0.2757 & 16.95 & 11.47 & 0.6749 & 0.73850 & 0.33021 & 0.38232 \\
\hline 0.2757 & 16.95 & 8.20 & 0.9385 & 0.73850 & 0.46596 & 0.38232 \\
\hline 0.1757 & 17.95 & 7.55 & 0.9923 & 1.07042 & 0.49284 & 0.49684 \\
\hline
\end{tabular}

TABLE 2

ARTIFICIAL NEURAL NETWORK SYNTHESIS RESULTS \& COMPARISON WITH TARGET

\begin{tabular}{|l|c|c|c|c|c|}
\hline $\mathbf{h}(\mathbf{c m})$ & $\mathbf{f r}(\mathbf{G H z})$ & $\mathbf{W}(\mathbf{c m})$ & $\mathbf{L}(\mathbf{c m})$ & -target & - RBF \\
\hline 0.1752 & 11.00 & 0.73850 & 0.38232 & 17.90 & 17.8905 \\
\hline 0.1752 & 10.70 & 0.73850 & 0.38232 & 17.90 & 17.8882 \\
\hline 0.1752 & 10.60 & 0.73851 & 0.38232 & 17.90 & 17.8770 \\
\hline 0.1752 & 9.27 & 0.73982 & 0.38271 & 17.90 & 17.8665 \\
\hline 0.1752 & 9.07 & 0.74181 & 0.38346 & 17.90 & 17.8648 \\
\hline 0.1752 & 7.00 & 1.07042 & 0.58056 & 17.90 & 17.8907 \\
\hline 0.1752 & 7.00 & 1.07042 & 0.58056 & 17.90 & 17.8907 \\
\hline 0.1752 & 11.00 & 0.73850 & 0.38232 & 17.90 & 17.8905 \\
\hline 0.2642 & 11.00 & 0.73850 & 0.38232 & 17.90 & 17.9549 \\
\hline 0.2752 & 9.55 & 0.73850 & 0.38232 & 17.90 & 17.9194 \\
\hline 0.1662 & 9.50 & 0.73850 & 0.38232 & 16.95 & 16.0291 \\
\hline 0.1662 & 11.30 & 0.73850 & 0.38232 & 16.95 & 16.9523 \\
\hline 0.2757 & 11.47 & 0.73850 & 0.38232 & 16.95 & 16.9154 \\
\hline 0.2757 & 8.20 & 0.73850 & 0.38232 & 16.95 & 16.9378 \\
\hline 0.1757 & 7.55 & 1.07042 & 0.49684 & 17.95 & 17.952 \\
\hline 0.1752 & 6.23 & 1.06530 & 0.57555 & 17.50 & 17.5011 \\
\hline 0.1752 & 10.23 & 0.73338 & 0.37731 & 17.50 & 17.4904 \\
\hline 0.2642 & 10.73 & 0.73338 & 0.37731 & 17.50 & 17.5548 \\
\hline 0.2752 & 9.28 & 0.73338 & 0.37731 & 17.50 & 17.5193 \\
\hline 0.1560 & 9.23 & 0.73338 & 0.37731 & 16.55 & 16.6290 \\
\hline 0.1560 & 11.03 & 0.73338 & 0.37731 & 16.55 & 16.5522 \\
\hline 0.2655 & 11.20 & 0.73338 & 0.37731 & 16.55 & 16.5153 \\
\hline 0.2655 & 7.93 & 0.73338 & 0.37731 & 16.55 & 16.5377 \\
\hline 0.1655 & 7.28 & 1.06570 & 0.49183 & 17.55 & 17.5519 \\
\hline & & & & & \\
\hline
\end{tabular}




\section{CONCLUSION}

We have designed micro strip antenna with the help of neural network which increased our system efficiency as well as making results more compatible with the standard values. Since, it is giving very accurate results therefore we have calculated different parameter of geometry by using this tool which are width of the patch and length of the patch, these values are obtained at the output of synthesized network when we input other parameter like height, resonant frequency and dielectric constant of substrate.

In this paper, the final stage of design procedure is analysis. Therefore, by reversing the parameter of synthesis that is input \& output data, we have determined analysis artificial neural network parameter. We have particular values of resonant frequency obtained from artificial neural network analysis and the same values are compared with the output of synthesis system and they are compared. In the present paper, we have formulated and design a micro strip patch antenna by using artificial neural network with the help of simplified general procedure.

\section{REFERENCES}

[1] Balanis C.A. (1997), Antenna Theory, John Wiley \& Sons, Inc.

[2] Bahl I.J. and Bhatia P. (1980), Micro strips Antennas, Dedham, MA, and Artech House.

[3] Pozar D.M. (1992), "Micro strip Antennas", Proc. IEEE, Vol. 80, pp.79-81, January.

[4] Angiulli G. and Versaci M. (May 2003), "Resonant frequency evaluation of micro strip antennas using a neural-fuzzy approach", Magnetics, IEEE Transactions, Vol. 39, No. 3, pp. 1333 - 1336.

[5] Devi S., Panda D.C. and Pattnaik S.S. (June 2002), “A novel method of using artificial neural networks to calculate input impedance of circular micro strip antenna", Antennas and Propagation Society International Symposium, Vol. 3, pp. 462 - 465, 16-21.

[6] G“uney K., Sa giro glu S. and Erler M. (Jan. 2002), "Generalized neural method to determine resonant frequencies of various micro strip antennas", International Journal of RF and Microwave Computer-Aided Engineering, Vol. 12, No. 1, pp. 131-139
[7] G"uney K. and Sarıkaya N. (April 2003), "Artificial neural networks for calculating the input resistance of circular micro strip antennas", Microwave and Optical Technology Letters, Vol. 37, No. 2, pp. 107-111

[8] Karabo־ga D., G“uney K. Sa giro־glu S. and Erler M. (April 1999), "Neural computation of resonant frequency of electrically thin and thick rectangular micro strip antennas", Microwaves, Antennas and Propagation, IEE Proceedings-Vol. 146, No. 2, pp. 155-159.

[9] Mishra R.K. and Patnaik A. (Dec. 1998), "Neural networkbased CAD model for the design of square- patch antennas", Antennas and Propagation, IEEE Transactions, Vol. 46, No. 12 , pp. $1890-1891$

[10] Mishra R.K. and Patnaik A. (Sept. 1999), "Neurospectral computation for input impedance of rectangular micro strip antenna", Electronics Letters, Vol. 35, No. 20, pp. 1691 1693.

[11] Mishra R.K. and Patnaik A. (Aug, 2003), "Designing rectangular patch antenna using the neuro spectral method", Antennas and Propagation, IEEE Transactions, Vol. 51, No. 8, pp. $1914-1921$.

[12] Park J. and Sandberg W. I. (1991), "Universal Approximation Using Radial Basis Function Networks", Neural Computation, Vol. 3, pp. 246-257.

[13] Sa giro־glu S., G̈uney K. and Erler M. (May 1999), "Calculation of bandwidth for electrically thin and thick rectangular micro strip antennas with the use of multilayered perceptron's", International Journal of RF and Microwave Computer-Aided Engineering, Vol. 9, No. 3, pp. 277-286.

[14] Sa giro glu S. and G"uney K. (1997), "Calculation of resonant frequency for an equilateral triangular micro strip antenna using artificial neural Networks", Microwave Opt. Technology Lett., Vol. 14, pp. 89-93.

[15] Sa giro glu S., G"uney K. and Erler M. (1998), "Resonant frequency calculation for circular micro strip antennas using artificial neural networks", International Journal of RF and Microwave Computer-Aided Engineering,Vol. 8, No. 3, pp. 270-277.

[16] Zhang Q. J. and Gupta K. C. (2000), Neural Networks for RF and Microwave Design, Artech House Publishers. 